casuística relevante. Neste domínio, a sua missão visa prestar Apoio Sanitário de área, com consultas de Medicina Dentária aos militares das Forças Armadas das Unidades Estabelecimentos e órgãos da região sul do país, e na sua capacidade sobrante aos seus familiares e a outros utentes devidamente enquadrados por subsistema de saúde protocolado, como forças de segurança; garantir as atividades de Saúde Operacional nomeadamente o apoio sanitário às ações de seleção de pessoal, de avaliação, de proteção e de promoção da saúde, e no aprontamento de Forças Nacionais Destacadas (FND). Em paralelo, desenvolver iniciativas de promoção e educação para a saúde oral no seio da comunidade. Os objetivos deste trabalho são caracterizar a atividade relativa à Medicina Dentária Militar desta Unidade de Saúde Militar de Évora, bem como a sua relevância nas vertentes operacional, assistencial e na relação com a comunidade. Materiais e métodos: Foi feita uma retrospetiva a 10 anos da estatística clínica deste Serviço de Medicina Dentária do Exército, no âmbito da Saúde Operacional e Assistencial, incluindo o aprontamento sanitário de 400 militares mobilizados para FND. Resultados: Neste período foram efetuadas 27.086 consultas, correspondentes a 41.983 tratamentos, 39\% dos quais de dentisteria, 21\% medicina dentária preventiva e Cirurgia Oral 15\%. No campo da saúde operacional, foi feito rastreio dentário de acordo com a sistematização da NATO a 420 militares que integraram FNDs em diversos teatros de operações, dos quais cerca de metade classes I e II, aptos, $45 \%$ como Classe III - Potencial baixa por motivos dentários e $5 \%$ de classe IV, indeterminados. A prevalência de cárie foi de 37\%. Conclusões: A Medicina Dentária Militar é uma especialidade com casuística relevante a nível assistencial e operacional, particularmente nesta Unidade. Demonstra como é exequível a constituição de serviços públicos de Medicina Dentária, e desempenha um papel ímpar na Saúde Operacional, contribuindo para um estado de saúde oral adequado para o potencial de combate e cumprimento das missões militares, particularmente das Forças Nacionais Destacadas.

http://doi.org/10.24873/j.rpemd.2019.12.562

\#101 Conhecimento sobre meios de prevenção da cárie dentária pelos pais de utentes pediátricos CrossMark

Rita Martinho Grão *, Maria del Rozario Garcillán, Susana Silva, Patrícia Nunes Correia

Universidad Complutense de Madrid, Universidade Católica Portuguesa Pólo de Viseu

Objetivos: Determinar o nível de conhecimento dos pais de utentes pediátricos sobre os meios de prevenção da cárie dentária e os seus hábitos de higiene oral em Portugal e em Madrid; estabelecer um estudo comparativo entre as duas amostras. Materiais e métodos: Utilização de um questionário em português e em castelhano numa amostra de conveniência proveniente das clínicas dentárias da Universidade Católica Portuguesa de Viseu e da Universidade Complutense de Madrid, bem como em clínicas de Coimbra, Viseu, Vale de Cambra e Madrid. Foi criada uma base de dados e os resultados foram analisados com o Software científico IBM-
-SPSS, versão 25. Os testes estatísticos utilizados para fazer o cruzamento das variáveis foram o teste do Qui-Quadrado, teste de Fisher e Correção de Continuidade, sendo utilizado um nível de significância estatística, $p<0,05$. Resultados: A amostra englobou um total de 154 questionários, 116 portugueses e 38 espanhóis, que responderam a questões sobre hábitos de higiene oral e prevenção, bem como perguntas sobre os dados sociodemográficos do inquirido e da criança. A maioria das crianças tinha idade igual ou superior a 6 anos ( $\mathrm{P}=96 \%$ vs $\mathrm{E}=97,4 \%)$. No âmbito dos hábitos de higiene oral, verificou-se que a maioria dos portugueses e espanhóis escova os dentes duas vezes ao dia $(P=72,4 \%$ vs $E=55,2 \%)$ $(p<0,05)$, embora exista um número maior de espanhóis que o faz três ou mais vezes ao dia $(34,2 \%)$ em comparação com os portugueses $(6,8 \%)(p<0,05)$. Consequentemente, verificou-se que a maior parte dos portugueses não escova os dentes à tarde $(92,2 \%)(p<0,05)$. Por fim, as crianças espanholas consultaram com mais frequência o médico dentista, concretamente de seis em seis meses ( $E=52,8 \%$ vs $P=32,5 \%$ ), enquanto que as portuguesas só o fizeram pelo menos uma vez por ano $(\mathrm{P}=57,9 \%$ vs $\mathrm{E}=44,4 \%)$. O motivo dessas consultas é maioritariamente para controlo dentário ( $\mathrm{P}=93,1 \%$ vs $\mathrm{E}=89,5 \%$ ). Conclusões: Ambas as populações estudadas mostraram um conhecimento satisfatório sobre os meios de prevenção da cárie dentária, embora não o apliquem na sua totalidade. Contudo, a amostra espanhola evidencia-se positivamente em algumas atitudes preventivas em comparação com a amostra portuguesa. Este trabalho evidencia a necessidade de continuar a implementar ações promotoras da saúde oral bem como a incentivar, desde cedo, a adesão a boas práticas de higiene oral.

http://doi.org/10.24873/j.rpemd.2019.12.563

\section{\#102 Comparação da resistência á tração de diferentes membranas de Fibrina Rica em Plaquetas}

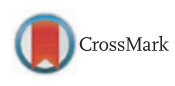

Martim Pascoal*, Nuno Bernardo Malta dos Santos, António Completo, Gustavo Vicentis de Oliveira Fernandes

Universidade de Aveiro, Universidade Católica Portuguesa Viseu

Objetivos: Este estudo teve por objectivo fazer comparação directa da resistência à tração entre membranas produzidas com diferentes protocolos de centrifugação, Leucocyte-Platelet Rich Fibrin (L-PRF) versus advanced-Platelet Rich Fibrin (a-PRF). Materiais e métodos: Após a colheita de sangue de uma pessoa saudável e sem histórico de toma de anticoagulantes ou outro medicamento, sob controlo alimentar, procedeu-se à confeção de membranas segundo os protocolos de L-PRF e a-PRF previamente descritos na literatura. De seguida, as membranas, $\mathrm{n}=26$ (13 para cada protocolo), foram submetidas a um teste mecânico de tração, para os quais foram obtidos valores de tração máxima e de tração média. A análise estatística dos dados foi feita com o teste t-Student não pareado. Resultados: Relativamente à tração média, o protocolo a-PRF e L-PRF, respetivamente, foram de 0.0288 N.mm-2 e 0.0192 N.mm-2 ( $p<0.05)$. Para a tração máxima 